\title{
Invasive Mucormycosis in a Patient With Liver Cirrhosis: Case Report and Review of the Literature
}

\author{
Hussien Elsiesy ${ }^{1,{ }^{*}}$, Mohamed Saad ${ }^{2}$, Mahmoud Shorman ${ }^{3}$, Samir Amr ${ }^{4}$, Faisal Abaalkhail ${ }^{1}$, \\ Almoutaz Hashim ${ }^{1}$, Waleed Al-Hamoudi ${ }^{1}$, Mohamed Al Sebayel ${ }^{1}$, Khalid Selim ${ }^{5}$ \\ 1 Department of Liver transplantation, King Faisal Specialist Hospital and RC, Riyadh, Saudi Arabia \\ 2 Department of Medicine, National Liver Institute, Menoufia, Egypt \\ 3 Department of Medicine, King Fahd Specialist Hospital-Dammam, Dammam, Saudi Arabia \\ 4 e \\ 5 Khalid Selim, Nazih Zuhdi Transplant Institute, INTEGRIS Baptist Medical Center, Oklahoma, USA \\ *Corresponding author: Hussien Elsiesy, Department of Liver transplantation, King Faisal Specialist Hospital and RC, Riyadh, Saudi Arabia. Tel: +96-6114424818, Fax: +96-6114424817, \\ E-mail: helsiesy@gmail.com.
}

Received: February 18, 2013; Revised: July 09, 2013; Accepted: July 21, 2013

\begin{abstract}
Introduction: Cutaneous Mucormycosis is a rare opportunistic infection caused by Zygomycetes class of fungi that is often fatal, requiring aggressive local control as well as systemic therapy. Few cases of mucormycosis were described in patients with liver cirrhosis, mostly rhino-orbital. To our knowledge, only two cases of upper extremity involvement was reported in cirrhosis while a few cases were reported in the post-transplant setting. We report herein the third case of upper extremity mucor infection in the setting of liver cirrhosis. Case Presentation: We described a rare case of forearm infection originating in a traumatic intravenous access portal in a 25 year-old woman with liver cirrhosis secondary to autoimmune hepatitis.

Discussion: She developed acute on chronic liver failure during the last trimester of pregnancy, which was terminated. Painful, erythematous lesion was noted on her right forearm in the area of intravenous access, which later became necrotic. Extensive debridement was done and histopathological examination confirmed the diagnosis of mucormycosis. The patient started on Amphotericin B. Her condition continued to deteriorate and ended up with above elbow amputation followed by right shoulder disarticulation. She died two days later due to multi-organ failure. In conclusion, forearm mucromycosis in liver cirrhosis can be fatal.
\end{abstract}

Keywords: Amputation; Gangrene; Liver Cirrhosis; Mucormycosis

\section{Introduction}

Mucormycosis is a rare opportunistic fungal infection characterized by infarction and necrosis of host tissues that is resulted from invasion of the vasculature by hyphae (1) The most common clinical presentation of mucormycosis is rhino-orbital-cerebral infection. It can also cause pulmonary, gastrointestinal, cutaneous, renal, and disseminated diseases (2). Diabetes is the most common risk factor, founded in 36\% of all cases, followed by hematologic malignancies (17\%), and solid organ or hematopoietic stem cell transplantation (12\%) (3). A systematic literature review of Medline was performed (01/1970-12/2012) including the search terms: Liver cirrhosis, Mucormycosis, amputation, gangrene. Additional manual review of the reference lists of the identified case reports. The medical literature on this subject revealed a small number of cases ( $\mathrm{n}=17$ cases including this case) reported in twelve papers (4-15), most with fatal outcome.

\section{Case Presentation}

A 25-year-old woman with liver cirrhosis secondary to autoimmune hepatitis diagnosed at a local hospital presented with fatigue, painless jaundice and lower limb swelling for two weeks, there was no evidence of hypertension or protenuria excluding the possibility of preeclampsia or eclampsia. There was no history of ascites, spontaneous bacterial peritonitis or hepatic encephathy. Pregnancy was terminated at 27th week at the local hospital. She was on $40 \mathrm{mg}$ of Prednisolone daily for possible autoimmune hepatitis, soon after, she developed steroid induced diabetes. She was referred to our hospital after 6 weeks of the intial presentation with two episodes of hematemesis, tachycardia (heart rate 120) and blood pressure of $84 / 50$. The patient was admitted to ICU and started on octreotide and Piperacillin/Tazobactam. Gastroscopy revealed gastric varices and bleeding was endoscopically controlled. During admission, liver function

Implication for health policy/practice/research/medical education:

This paper is a case report with review of the literature collecting all cases of mucormycosis in patients with liver cirrhosis, the message this paper is conveying is that even though mucormycosis is rare in the setting of cirrhosis, but it carries a very high mortality compared with mucormycosis in the setting of diabetes or hematological malignancies. This will be of an interest to the general and transplant hepatologist.

Copyright (C2013, Kowsar Corp.; Licensee Kowsar Ltd. This is an Open Access article distributed under the terms of the Creative Commons Attribution License(http:/ creativecommons.org/licenses/by/3.0), which permits unrestricted use, distribution, and reproduction in any medium, provided the original work is properly cited. 
tests worsened, and transjugular liver biopsy showed established liver cirrhosis with no obvious active pathology. We started tapering the steroids slowly after being on $40 \mathrm{mg}$ for 4 weeks with no improvement of jaundice without evidence of active inflammation on liver biopsy. During admission, she developed painful, erythematous lesion on her right forearm at the IV access area that became necrotic and spread quickly and increased in size over a period of 24 hours. The lesion included the skin and subcutaneous tissue and was flecked with tiny black spots. Vital signs: temp $37.4^{\circ} \mathrm{C}, \mathrm{BP} 128 / 80 \mathrm{mmHg}$, pulse 66 /min, RR 20 /min, SaO2 100\% on room air.

Chest was clear to auscultation and cardiovascular exam was normal. Abdomen was soft and lax, distended with ascites but no tenderness. Extremities showed mild lower limb edema. Her investigations showed a white cell count $14.24 \times 103 / \mathrm{mm} 3$ with Neutrophils of $86 \%$, hemoglobin $9.2 \mathrm{~g} / \mathrm{dl}$, and platelets of $103000 / \mathrm{mm} 3$, LFT: total bilirubin $457 \mathrm{umol} / \mathrm{L}$, direct 321, alkaline phosphatase 430U/L, ALT 174U/L, AST 248U/L, gamma GT 227, total protein 51g/L, albumin 18g/L, PT 19.4 and INR was 1.7, PTT 58 , renal function test was within normal, fasting glucose $9.8 \mathrm{mmol} / \mathrm{L}$. ESR was 2, Blood culture showed no growth after 5 days.

MRI of the right upper extremity showed inflammatory changes through the anterior compartment of the forearm suggestive of fasciitis.

She was taken to the operating room for debridement after 24 hours, delay was due to anesthesia issues in this high risk patients. At this point after 18 days of admission, Prednisolone dose reached $20 \mathrm{mg}$ daily and antibiotic changed from Piperacillin/Tazobactam to Imipenem when the culture results from tissue biopsy grew Klebsiella Pneumonia extended spectrum b lactamase producer (ESBL).

Histopathological examination of the debrided soft tissue revealed necrotic subcutaneous fat and skeletal muscle fibers invaded by broad hyphae, irregularly branched, with rare septations suggestive of mucormycosis (Figure $1 \mathrm{~A}$ and $1 \mathrm{~B}$ ). There were no clinical symptoms or signs of visceral dissemination of infection to warrant further imaging or biopsy. The patient started on Amphotericin B (Abelcet $($ ) 300mg IV OD after 20 days of admission and was continued on imipenem. In spite of surgical debridement, her condition continued to deteriorate and ended up after 6 days with above elbow amputation, tissue-cultures for both bacteria and fungi were negative. Amphotericin B was stopped after amputation as it was considered that the source of infection was eliminated but was restarted again 3 days after discontinuation with imipenem continued all through with the addition of vancomycin. The patient continued to deteriorate and the stump showed signs of disseminated infection, which proved to be invasive mucormycosis infection. Right shoulder disarticulation done but she passed away 2 days later due to multi-organ failure after 35 days in the hospital.
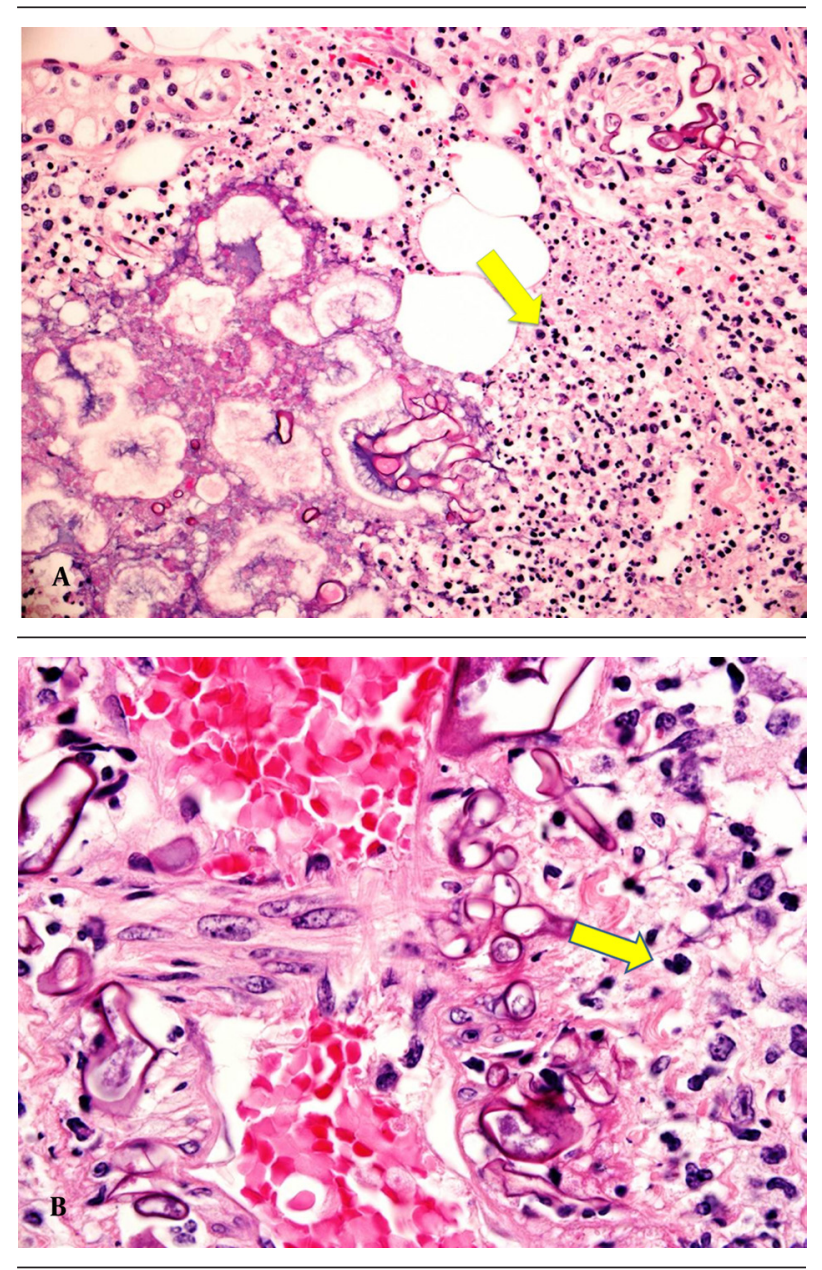

Figure 1. A) Extensive fat necrosis from soft tissue of forearm with neutrophilic infiltrate and several irregular broad fungal hyphae. (PAS stain x200) B) Irregular thick fungal hyphae with rare septa invading the walls of blood vessels, a feature of mucor fungal infection. (PAS stain $\mathrm{x} 400$ )

\section{Discussion}

There are more than 900 cases of mucormycosis reported in the literature (3), Diabetes is the most common risk factor but it has been described with hematological malignancy, solid tumors, bone marrow and solid organ transplantation and recipients of deferoxamine therapy (3). Sixteen cases of mucormycosis are reported in patients with liver cirrhosis (4-15). The majority of these cases are rhino-orbital. Three cases including this case were upper extremity and one case was gastric involvement (Table 1). 


\begin{tabular}{|c|c|c|c|c|c|c|c|c|c|}
\hline $\begin{array}{l}\text { Pa- } \\
\text { tients } \\
\text { no }\end{array}$ & $\begin{array}{l}\text { Age/ } \\
\text { Gen- } \\
\text { der }\end{array}$ & $\begin{array}{l}\text { Child } \\
\text { Pugh } \\
\text { class }\end{array}$ & $\begin{array}{l}\text { Eti- } \\
\text { ol- } \\
\text { ogy }\end{array}$ & $\begin{array}{l}\text { Cirrho- } \\
\text { sis }\end{array}$ & $\begin{array}{l}\text { Comor- } \\
\text { bidity } \\
\text { Com- } \\
\text { plica- } \\
\text { tion }\end{array}$ & Involved Area & Treatment & $\begin{array}{l}\text { Out- } \\
\text { come }\end{array}$ & $\begin{array}{l}\text { Ref- } \\
\text { er- } \\
\text { ence }\end{array}$ \\
\hline $\mathbf{1}$ & $\begin{array}{l}44 / \mathrm{Fe}- \\
\text { male }\end{array}$ & B & $\mathrm{NR}^{\mathrm{a}}$ & NR & $\mathrm{LC}^{\mathrm{a}}$ & Ethmoid sinus, orbit & None & Died & (9) \\
\hline \multicolumn{10}{|l|}{1993} \\
\hline 2 & $\begin{array}{l}53 / \\
\text { Male }\end{array}$ & B & $\mathrm{HCV}^{\mathrm{a}}$ & $\mathrm{HCC}^{\mathrm{a}}$ & $\mathrm{LC}, \mathrm{DM}^{\mathrm{a}}$ & Not done & None & Died & $(10)$ \\
\hline \multicolumn{10}{|l|}{1998} \\
\hline 3 & $58 / \mathrm{F}$ & $\mathrm{C}$ & NR & NR & $\begin{array}{l}\mathrm{LC}, \mathrm{DM}, \\
\mathrm{RI}^{\mathrm{a}}\end{array}$ & $\begin{array}{l}\text { Paranasal sinuses, orbit, } \\
\text { cerebral }\end{array}$ & Liposomal AmB ${ }^{a}$ & Died & (7) \\
\hline \multicolumn{10}{|l|}{2003} \\
\hline 4 & $39 / \mathrm{M}$ & B & $\mathrm{HCV}$ & None & LC, DM & $\begin{array}{l}\text { Maxillary, ethmoid \& sphe- } \\
\text { noid sinus, cerebral }\end{array}$ & Surgery/AmB & Died & $(4)$ \\
\hline \multicolumn{10}{|l|}{2007} \\
\hline 5 & $57 / \mathrm{M}$ & $\mathrm{C}$ & $\mathrm{HCV}$ & $\begin{array}{l}\text { PSE }^{a} \\
\text { SBPa } \\
\text { HCC }\end{array}$ & LC & Not done & $\mathrm{AmB}$ & Died & $(4)$ \\
\hline \multicolumn{10}{|l|}{2007} \\
\hline 6 & $55 / M$ & $\mathrm{C}$ & HBV & $\begin{array}{l}\text { PSE, SBP, } \\
\text { HRS } \\
\text { HCC }^{a}\end{array}$ & LC & Maxillary, and ethmoid sinus & None & Died & $(4)$ \\
\hline \multicolumn{10}{|l|}{2007} \\
\hline 7 & $15 / F$ & $\mathrm{C}$ & $\mathrm{AIH}$ & PSE, SBP & $\begin{array}{l}\text { LC, DM, } \\
\text { steroid } \\
\text { therapy }\end{array}$ & Maxillary sinus & None & Died & (4) \\
\hline \multicolumn{10}{|l|}{2007} \\
\hline 8 & $53 / \mathrm{M}$ & $\mathrm{C}$ & $\mathrm{HCV}$ & None & LC, DM & $\begin{array}{l}\text { Maxillary, ethmoid and sphe- } \\
\text { noid sinuses, orbit, cerebral }\end{array}$ & $\begin{array}{l}\text { Surgery (Orbit } \\
\text { enucleation \& endo- } \\
\text { scopic debridement } \\
\text { of sinuses)/ AmB }\end{array}$ & Died & (4) \\
\hline
\end{tabular}

\begin{tabular}{|c|c|c|c|c|c|c|c|c|c|}
\hline \multicolumn{10}{|c|}{2007} \\
\hline 9 & $35 / M$ & C & $\mathrm{HCV}$ & SBP, HRS & LC, DM & $\begin{array}{l}\text { Maxillary ethmoid and sphe- } \\
\text { noid sinus, orbit }\end{array}$ & $\mathrm{AmB}$ & Died & (4) \\
\hline \multicolumn{10}{|c|}{2007} \\
\hline 10 & $63 / \mathrm{F}$ & B & $\mathrm{HCV}$ & None & LC & $\begin{array}{l}\text { Maxillary, ethmoid sphenoid } \\
\text { sinuses and orbit }\end{array}$ & $\begin{array}{l}\text { Surgery/AmB, Lipo- } \\
\text { somal AmB }\end{array}$ & Alive & (12) \\
\hline \multicolumn{10}{|c|}{2009} \\
\hline 11 & $42 / \mathrm{M}$ & $\mathrm{C}$ & HBV & PSE & None & $\begin{array}{l}\text { Sphenoid, ethmoid, and } \\
\text { maxillary sinuses }\end{array}$ & $\mathrm{AmB}$ & Died & (5) \\
\hline \multicolumn{10}{|c|}{2010} \\
\hline 12 & $59 / \mathrm{F}$ & C & $\begin{array}{l}\text { Alco- } \\
\text { hol }\end{array}$ & PSE & LC, HTN & $\begin{array}{l}\text { Paranasal sinuses, orbits, } \\
\text { bilateral infratemporal fossa, } \\
\text { right cavernous sinuses, } \\
\text { bilateral optic nerve }\end{array}$ & & Died & (6) \\
\hline \multicolumn{10}{|c|}{2011} \\
\hline 13 & $65 / M$ & B & $\mathrm{HCV}$ & None & $\begin{array}{l}\text { DM, } \\
\text { HTN }\end{array}$ & $\begin{array}{l}\text { Maxillary and ethmoid } \\
\text { sinuses }\end{array}$ & $\begin{array}{l}\text { Surgery, AmB, } \\
\text { liposomal AmB, itra- } \\
\text { conazole, posacon- } \\
\text { azole }\end{array}$ & Alive & (11) \\
\hline
\end{tabular}


Elsiesy H et al.

\begin{tabular}{|c|c|c|c|c|c|c|c|c|c|c|}
\hline \multicolumn{11}{|c|}{2012} \\
\hline 14 & $47 / \mathrm{M}$ & $\mathrm{C}$ & $\mathrm{HCV}$ & $\begin{array}{l}\text { Gastric } \\
\text { mucor- } \\
\text { mycosis, } \\
\text { Cirrhosis }\end{array}$ & $\begin{array}{l}\text { HTN, } \\
\text { DM }\end{array}$ & Gastric Ulcer & Amphotericin B & Died & $(14)$ & \\
\hline \multicolumn{11}{|c|}{2012} \\
\hline 15 & $38 / \mathrm{F}$ & $\mathrm{C}$ & $\begin{array}{l}\text { Alco- } \\
\text { hol }\end{array}$ & $\begin{array}{l}\text { PSE, SBP, } \\
\text { HRS }\end{array}$ & $\begin{array}{l}\text { LC, ste- } \\
\text { roids }\end{array}$ & Right Forearm & Amputation & Died & $(13)$ & \\
\hline \multicolumn{11}{|c|}{2007} \\
\hline 16 & $48 / \mathrm{F}$ & NR & $\begin{array}{l}\text { Alco- } \\
\text { hol }\end{array}$ & PSE & NR & Right Forearm & Local excision & Died & $(15)$ & \\
\hline \multicolumn{11}{|c|}{2010} \\
\hline 17 & $25 / \mathrm{F}$ & $\mathrm{C}$ & $\mathrm{AIH}$ & $\begin{array}{l}\text { Variceal } \\
\text { bleed- } \\
\text { ing }\end{array}$ & $\begin{array}{l}\text { LC, ste- } \\
\text { roids }\end{array}$ & Right Forearm & Amputation, AmB & Died & $\begin{array}{l}\text { This } \\
\text { case }\end{array}$ & 2013 \\
\hline
\end{tabular}

\footnotetext{
a Abbreviations: AmB, amphotericin B; DM, diabetes mellitus; HBV, Hepatitis B virus; HCV, Hepatitis C virus; HCC, hepatocellular carcinoma; HTN, hypertension; PSE, portosystemic encephalopathy; SBP, spontaneous bacterial peritonitis; HRS, hepatorenal syndrome; LC, liver cirrhosis; RI, renal insufficiency; NR, Not reported
}

To our knowledge, there are only two cases of upper limb mucormycosis which are reported previously in association with cirrhosis $(13,15)$. Moreover, cases of limb involvement in the post-transplant setting are rare (16). Raizman et al., reported in 2007 the first case of upper extremity mucormycosis in the setting of liver cirrhosis in a 38 year old lady with alcoholic cirrhosis, who was on steroids. She developed gangrene and underwent amputation. The diagnosis was confirmed after the patient death (13). Wollstein et al, reported another case in 2010 of a 48 year old lady with decompensated alcoholic cirrhosis and hepatic encephalopathy admitted with pneumonia and septic shock, developed of right forearm mucormycosis treated with local excision, and eventually she died (15). In our case, the diagnosis was made relatively early and the patient was started on antifungal therapy few days after the initial presentation. As in the case reported in 2007, our patient died despite above elbow amputation followed by shoulder disarticulation together with antifungal therapy. There was an initial insult to the forearm with an IV access in our case. In some cases a traumatic incident preceded the infection. In a case described by Raizman et al., the infection seemed to have started in an arterial line and caused necrosis of the hand distally (13). Another case of localized mucormycosis was described following an intramuscular injection of corticosteroids (17). Another case was reported after use of contaminated dressing (18).

Mucormycosis in liver cirrhosis has been reported, including the present case, in 17 patients: 9 males and 8 females. Cirrhosis was due to $\mathrm{HCV}$ in 8 patients; $\mathrm{HBV}$ in 2; autoimmune hepatitis (AIH) in 2; and alcoholic liver disease (ALD) in 3. The etiology was not reported in 2 cases. Mucormycosis was not reported in Child A, there were 11 cases with Child C and 5 with Child B and in one case, the Child's score was not reported (15). The two survivals of mucormycosis in patients with liver cirrhosis were in pa- tients with Child' B cirrhosis $(11,12)$. Eight cases have DM as co-morbidity while 3 patients were on steroids.

The mortality is high despite aggressive surgical debridement and proper antifungal and antibacterial coverage, there was a relative delay in diagnosing the fungal infection but treatment started once the cultures came back positive. The outcome may be determined mainly by underlying risk factor; mucormycosis in diabetics has $60 \%$ to $90 \%$ the survival, where it is $20 \%-50 \%$ in Leukemia. In Cirrhosis it is $11.7 \%$ ( 2 out of 17 patients).

In conclusion; mucormycosis carries high mortality in patients with liver cirrhosis despite aggressive treatment, the three cases with limb involvement, the gastric mucromycosis and 11 out of 13 cases of rhino-orbital involvement died. From this case and in reviewing the literature, it seems that underlying risk factors of the patient determine the final outcome more than the particular treatment provided.

\section{Acknowledgements}

We acknowledge Juvimar Arpon for providing technical help.

\section{Authors Contribution}

Hussien Elsiesy: writing and revising the manuscript, reviewing the literature. Mohamed Saad: wrote the case report. Mahmoud Shorman: wrote/edited the medical therapy part. Samir Amr: wrote the pathology part. Faisal Abaalkhail, Almoutaz Hashim and Waleed Al Hamoudi reviewed the literature. Mohamed Al Sebayel and Khalid Selim constructed the table and helped in the final editing.

\section{Financial Disclosure}

Dr. Elsiesy reported receiving honoraria for speaking from JSK, Roche, BMS. 


\section{Funding/Support}

No funding needed for this manuscript

\section{References}

1. Mantadakis E, Samonis G. Clinical presentation of zygomycosis. Clinical Microbiology and Infection. 2009;15:15-20.

2. Kauffman C, Malani A. Zygomycosis: An emerging fungal infection with new options for management. Current Infectious Disease Reports. 2007;9(6):435-40.

3. Roden MM, Zaoutis TE, Buchanan WL, Knudsen TA, Sarkisova TA, Schaufele RL, et al. Epidemiology and outcome of zygomycosis: a review of 929 reported cases. Clin Infect Dis. 2005;41(5):634-53.

4. Abbas Z, Jafri W, Rasool S, Abid S, Hameed I. Mucormycosis in patients with complicated cirrhosis. Singapore Med J. 2007;48(1):6973.

5. Ataseven H, Yuksel I, Gultuna S, Koklu S, Uysal S, Basar O, et al. Fatal rhinocerebral mucormycosis under the shade of hepatic encephalopathy. Ann Hepatol. 2010;9(4):462-4.

6. Chaudhry A, Hirano SA, Hayes TJ, Torosky C. Fatal rhino-orbitocerebral mucormycosis in a patient with liver disease. J Am Acad Dermatol. 2011;65(1):241-3.

7. Georgopoulou S, Kounougeri E, Katsenos C, Rizos M, Michalopoulos A. Rhinocerebral mucormycosis in a patient with cirrhosis and chronic renal failure. Hepatogastroenterology. 2003;50(51):843-5.

8. Hibbett DS, Binder M, Bischoff JF, Blackwell M, Cannon PF, Eriksson $\mathrm{OE}$, et al. A higher-level phylogenetic classification of the Fungi. Mycol Res. 2007;111(Pt 5):509-47.

9. Hofman P, Gari-Toussaint M, De Bievre C, Michiels JF, d'Horpock FA, Loubiere R. [Rhino-orbito-cerebral mucormycosis caused by
Rhizopus oryzae.A typical case in a cirrhotic patient]. Ann Pathol. 1993;13(3):180-3.

10. Kikuchi H, Kinoshita Y, Arima K, Doh-ura K, Hisatomi Y, Hashimoto T, et al. An autopsy case of rhino-orbito-cerebral mucormycosis associated with multiple cranial nerve palsy and subsequent subarachnoid hemorrhage]. Rinsh shinkeigaku=Clinical neurology. 1998;38(3):252.

11. Lin SY, Lu PL, Tsai KB, Lin CY, Lin WR, Chen TC, et al. A mucormycosis case in a cirrhotic patient successfully treated with posaconazole and review of published literature. Mycopathologia. 2012;174(5-6):499-504.

12. Pellicelli AM, D'Ambrosio C, Villani R, Cerasari G, Ialongo P, Cortese A, et al. Liver cirrhosis and rhino-orbital mucormycosis, a possible but rare association: description of a clinical case and literature review. Braz J Infect Dis. 2009;13(4):314-6.

13. Raizman NM, Parisien M, Grafe MW, Gordon RJ, Rosenwasser MP. Mucormycosis of the upper extremity in a patient with alcoholic encephalopathy. J Hand Surg Am. 2007;32(3):384-8.

14. Rudler M, Barret M, Poynard T, Thabut D. Gastric mucormycosis: A rare cause of gastrointestinal bleeding in cirrhosis. Clinics and research in hepatology and gastroenterology. 2012;36(2):e32-e3.

15. Wollstein R, Palekar A. Mucormycosis infection following intravenous access in the forearm. The Canadian Journal of Plastic Surgery. 2010;18(2):e30.

16. Tobon AM, Arango M, Fernandez D, Restrepo A. Mucormycosis (zygomycosis) in a heart-kidney transplant recipient: recovery after posaconazole therapy. Clin Infect Dis. 2003;36(11):1488-91.

17. Jain JK, Markowitz A, Khilanani PV, Lauter CB. Localized mucormycosis following intramuscular corticosteroid. Case report and review of the literature. Am J Med Sci.1978;275(2):209-16.

18. Everett ED, Pearson S, Rogers W. Rhizopus surgical wound infection with elasticized adhesive tape dressings. Arch Surg. 1979;114(6):738-9. 
